\title{
Factores de riesgo para cáncer gástrico: ¿cuál es su papel?
}

\section{Risk factors for gastric cancer. What is their role?}

Ricardo Oliveros-Wilches, ${ }^{1 *}$ (1) Helena Facundo-Navia, ${ }^{1}$ (D) Ana Deise Bonilla, ${ }^{1}$ (D) Raúl Pinilla-Morales. ${ }^{1}$

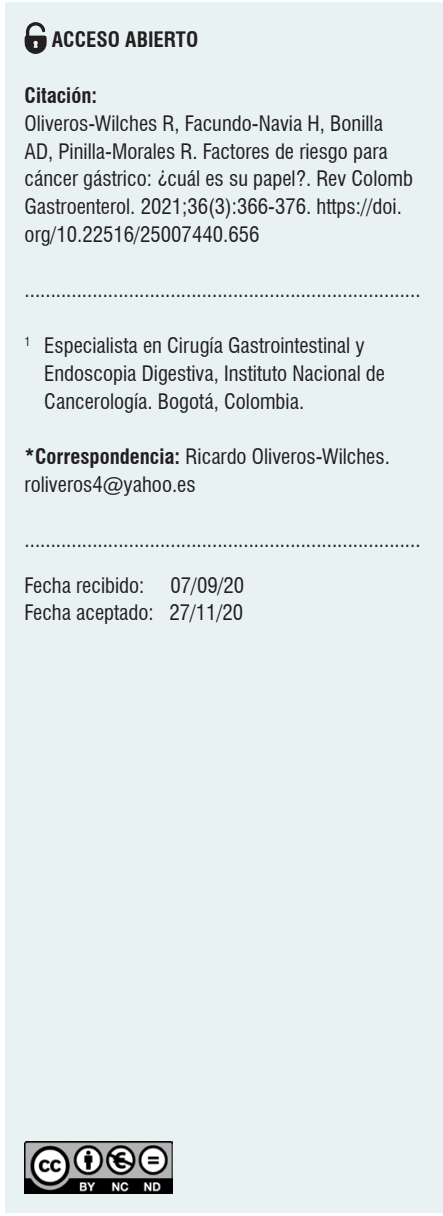

\section{Resumen}

El proceso de la carcinogénesis gástrica es multifactorial y secuencial. No lo comprendemos completamente, pero conocemos la historia natural de la enfermedad descrita por el Dr. Pelayo-Correa y también la existencia de múltiples factores de riesgo. La identificación de estos factores que participan en cada paso y el manejo apropiado de los mismos podría ayudar a reducir la incidencia del cáncer gástrico (CG). Probablemente, la infección por Helicobacter pylori (HP) es el factor de riesgo más conocido y discutido; sin embargo, existen otros factores como los relacionados con el estilo de vida, tipo de dieta, historia familiar de cáncer, entre otros, que también participan en el desarrollo e incidencia de este tipo de cáncer y tienen una amplia ventana de tiempo para ejercer su influencia.

A nivel poblacional, la identificación y el conocimiento de estos factores de riesgo puede proporcionar un entendimiento en la etiología de la enfermedad y es esencial para planear, monitorizar y evaluar los planes, políticas y estrategias de prevención. Es necesario, por lo tanto, desarrollar una herramienta de estudio basada en la identificación de factores de riesgo que se pueda sumar a los hallazgos endoscópicos e histológicos y ser usada en la práctica clínica para la clasificación del riesgo de CG.

\section{Palabras clave}

Cáncer gástrico, factores de riesgo, prevención, grupos de alto riesgo.

\section{Abstract}

The process of gastric carcinogenesis is multifactorial and sequential. We do not fully understand this, but we know the natural history of the disease described by Dr. Pelayo Correa and the existence of multiple risk factors. Identifying the factors involved in each step and managing them appropriately could help reduce the incidence of gastric cancer (GC). Helicobacter pylori (HP) infection is probably the most widely known and discussed risk factor. However, there are other factors such as lifestyle, type of diet, family history of cancer, among others, that are also involved in the development and incidence of this cancer and have a wide window of time to exert their influence.

At the population level, identification and awareness of these risk factors can provide insight into the etiology of the disease and are essential for planning, monitoring and evaluating prevention plans, policies and strategies. It is therefore necessary to develop a study tool based on risk factor identification that can be added to endoscopic and histological findings and used in clinical practice for GC risk classification.

\author{
Keywords \\ Gastric cancer; Risk factors; Prevention; High-risk groups.
}




\section{INTRODUCCIÓN}

Cuando aparecen los signos y síntomas clínicos de una enfermedad, la intervención curativa es poco posible y revertir la enfermedad es improbable. Hasta la fecha, la medicina preventiva mundial se ha centrado en la prevención primaria, cuyos objetivos generales son evitar la aparición de la enfermedad promoviendo hábitos saludables y controlando los factores de riesgo ${ }^{(1)}$. Otro enfoque, quizá más útil en patologías específicas como el cáncer, es identificar activamente individuos con alto riesgo de desarrollar la enfermedad e intervenirlos con estrategias preventivas ${ }^{(2)}$.

En el quehacer médico y quirúrgico, el cáncer gástrico (CG) es una enfermedad que nos sigue desafiando. A pesar de la disminución global en su incidencia, permanece como un problema serio de salud y es comunmente diagnosticado en estadios avanzados ${ }^{(3)}$.

Cada vez que se diagnostica un paciente con CG avanzado, las probabilidades de ser llevado a cirugía con intención curativa son muy bajas, al igual que su supervivencia a los 5 años. En ese gran grupo de pacientes hay quienes nunca consultaron, quienes consultaron por síntomas gastrointestinales inespecíficos y recibieron un diagnóstico clínico de "gastritis" con el manejo sintomático que suele acompañarlo; sin embargo, también curiosa y tristemente hasta una cuarta parte de los pacientes con diagnóstico reciente de CG habían sido llevados a un estudio endoscópico en los 3 años previos $^{(3)}$.

Para el entendimiento de una enfermedad es muy importante conocer su historia natural. El Dr. Pelayo-Correa describió la progresión de la mucosa normal al CG como un proceso por pasos ${ }^{(4)}$, con un intervalo extremadamente largo entre el comienzo de la gastritis y la malignización. Todo lo anterior proporciona tiempo y oportunidad para interrumpir la cascada oncogénica y constituye una justificación razonable para el desarrollo de estrategias de prevención primarias y secundarias ${ }^{(5)}$.

No todos los CG obedecen a esta secuencia, y sabemos que también se ha descrito una progresión mucho más rápida para algunos cánceres del tipo difuso, particularmente en personas jóvenes y con susceptibilidad genética $^{(6)}$. Sin embargo, la secuencia de Pelayo-Correa ${ }^{(4)}$ sigue explicando la mayoría de los CG. Identificamos entonces un primer desafío que se refiere a la calidad de los estudios endoscópicos rutinarios y su rendimiento en la identificación de lesiones premalignas y cánceres tempranos.

Además, las decisiones clínicas se toman con base en los informes endoscópicos e histopatológicos que, la mayoría de las veces, no buscan activamente condiciones premalignas y se limitan a tomar biopsias del antro, olvidando la historia natural de la enfermedad y no clasificando el riesgo real para CG ni su seguimiento pertinente ${ }^{(7)}$. Un entrena- miento consciente y dirigido a la evaluación de cambios sutiles de la mucosa muy probablemente incrementaría el diagnóstico de lesiones tempranas.

Conocemos que el proceso de la carcinogénesis es multifactorial y secuencial, y es bien sabido que no todos los sujetos con lesiones preneoplásicas llegarán a desarrollar CG, pero otros tantos sí lo harán. En ese proceso complejo, que aún no acabamos de comprender, intervienen también múltiples factores de riesgo. Uno de ellos es cualquier rasgo, característica o exposición de un individuo que aumenta la probabilidad de sufrir una enfermedad o lesión. La identificación de los factores de riesgo que participan en cada paso y el manejo apropiado de los mismos podría ayudar a reducir la incidencia del CG. A nivel poblacional, el conocimiento de estos factores de riesgo es esencial para planear, monitorizar y evaluar los planes, políticas y estrategias para el control de este cáncer ${ }^{(8)}$.

Probablemente, el factor de riesgo más conocido y discutido es la infección por Helicobacter pylori (HP), cuya erradicación ha reducido la incidencia del CG. Actualmente se considera que la mejor opción para reducir la mortalidad por esta enfermedad es la estrategia de erradicación en combinación con programas de seguimiento endoscópico para individuos de alto riesgo, que permitan la adecuada identificación y tratamiento de lesiones tempranas ${ }^{(5)}$. Sin embargo, existen otros factores directamente relacionados con el estilo de vida que también participan en el desarrollo e incidencia de este tipo de cáncer y que, como hemos dicho, tienen una amplia ventana de tiempo para ejercer su influencia ${ }^{(8,9)}$ (Figura 1).

Las diferentes guías de manejo para la prevención y detección temprana del CG se centran en la identificación de lesiones y condiciones premalignas gástricas confirmadas en los hallazgos histopatológicos, y de acuerdo con su gravedad y extensión proponen un seguimiento. En cambio, los factores de riesgo epidemiológicos no tienen un peso definido al momento de establecer grupos de alto o bajo riesgo ${ }^{(10)}$.

En este momento, la pregunta sugerida es si un paciente obeso, fumador, que consume alcohol y tiene antecedentes familiares de CG debe tener el mismo intervalo de seguimiento que un paciente atlético, con buenos hábitos alimentarios y sin antecedentes familiares de cáncer, pero con los mismos hallazgos endoscópicos e histopatológicos.

Las estrategias encaminadas a construir un estilo de vida que no favorezca el cáncer tienen el potencial de ser aplicables masivamente en la población y de impactar a las personas desde la juventud. Creemos que es necesario desarrollar una herramienta de estudio basada en la identificación de factores de riesgo que se pueda sumar a los hallazgos endoscópicos e histológicos y ser usada en la práctica clínica para la clasificación del riesgo de $\mathrm{CG}^{(11)}$. 


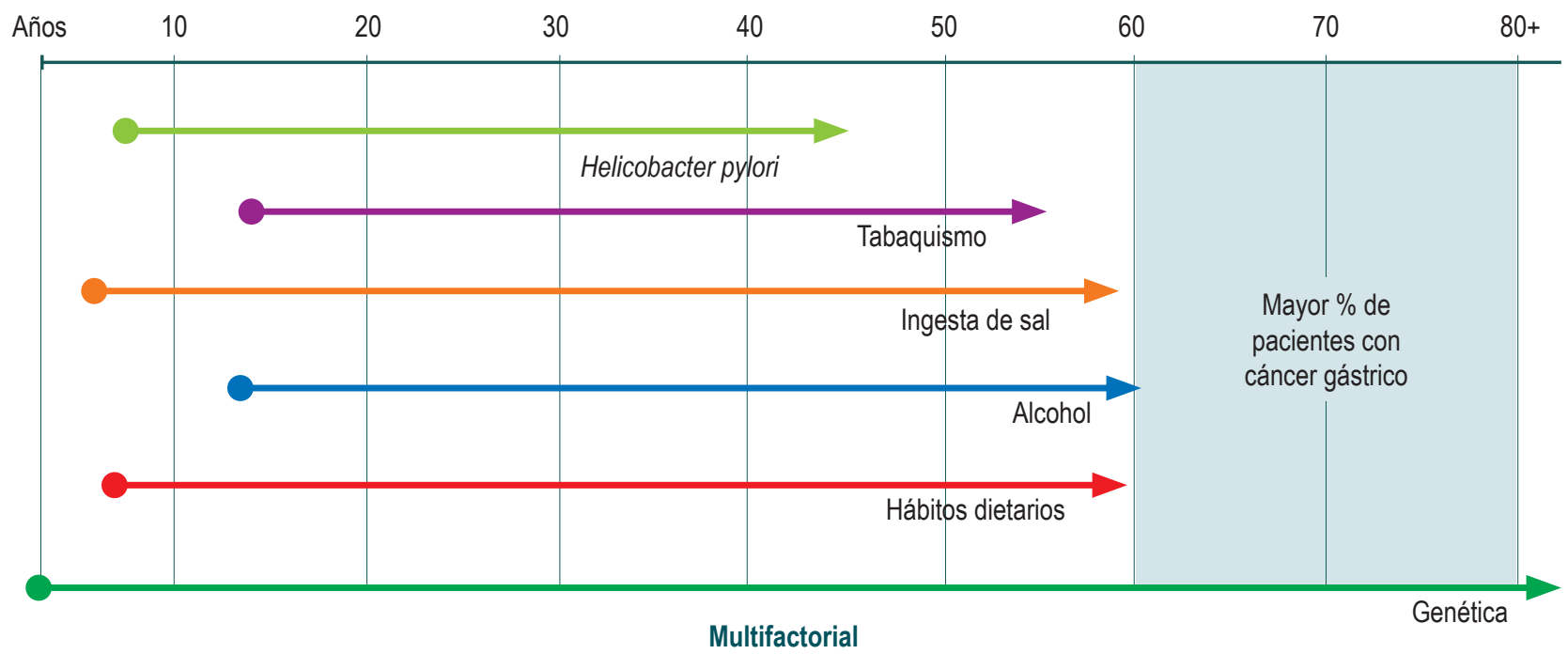

Figura 1. Cáncer gástrico. Factores de riesgo en el tiempo (historia natural). Elaboración propia.

\section{APORTE EPIDEMIOLÓGICO - FACTORES DE RIESGO}

Se han estudiado y descrito diferentes factores que incrementan el riesgo de CG o que lo reducen (protectores) ${ }^{(9)}$. Los factores de riesgo han sido clasificados en no modificables y modificables: dentro de los primeros están la edad avanzada, el género masculino, la etnia y los aspectos genéticos; entre los modificables están el consumo de una dieta no saludable, el tabaquismo, la ingesta de alcohol, la ingesta de sal y la infección por el HP. Estos últimos, por ser modificables, deben ser el foco de atención cuando se diseñan programas de prevención ${ }^{(9,12,13)}$.

El desafío en este punto es identificar e investigar los factores de riesgo para CG con valor estadísticamente significativo. Existen múltiples trabajos que describen en qué magnitud se aumenta o disminuye el riesgo de CG con relación a cada factor ${ }^{(8,9)}$.

Yusefi y colaboradores ${ }^{(8)}$, en una revisión sistemática de 43 estudios, identificaron 52 factores de riesgo clasificados en: dietarios, estilo de vida, predisposición genética más historia familiar, infecciosos, características demográficas, exposición ocupacional y radiación ionizante. Los autores reportaron una asociación entre la infección por HP y CG con un Odds ratio (OR) de 3 (intervalo de confianza [IC] del $95 \% 2,42$ a 3,72). 12 de los trabajos revisados en este mismo estudio identificaron al tabaquismo como factor de riesgo para CG y es así reconocido por la Agencia Internacional para la Investigación del Cáncer (IARC por sus siglas en inglés).

La asociación causal entre el HP y el CG está firmemente establecida por estudios epidemiológicos y clínicos. El
CG se desarrolla únicamente en el $1 \%$ de los pacientes infectados, pero al mismo tiempo más del $90 \%$ de los pacientes con CG tienen o han tenido infección por esta bacteria, de modo que el HP es el principal factor de riesgo para el $\mathrm{CG}^{(3)}$. Esta infección es una de las más comunes en el humano y afecta a más del $50 \%$ de la población en el mundo; puede ser adquirida en la infancia, su prevalencia está directamente relacionada con la edad y la mayoría de los casos son asintomáticos ${ }^{(8,13)}$. Muchos países con una alta infección por HP no tienen una alta incidencia de CG. La interacción entre el HP, la genética y la dieta pueden explicar estas discrepancias ${ }^{(14)}$.

En un metaanálisis ${ }^{(15)}$ de 19 estudios de cohortes o casos y controles, que incluyó 2491 pacientes y 3959 controles, el OR para CG en pacientes con infección por HP, diagnosticado serológicamente, fue reportado como 1,92 (IC del95\% $1,32$ a 2,78$)$.

El HP actúa indirectamente sobre las células epiteliales gástricas causando inflamación y de forma directa sobre las mismas, modulando su función a través de agentes de la bacteria como el Cag $\mathrm{A}^{(9,13)}$.

Un estudio de casos y controles del Reino Unido estimó que el $22 \%$ de los casos con CG están relacionados con el tabaquismo y el $32 \%$ tienen relación con el $\mathrm{HP}^{(8,9)}$.

El consumo de bebidas alcohólicas es otro factor de riesgo que ha sido evaluado con resultados no homogéneos. Este hallazgo fue reportado en 7 de los estudios incluidos en el reporte de Yusefi y colaboradores ${ }^{(8)}$. En su metaanálisis, Ma y colaboradores ${ }^{(16)}$ encontraron que el consumo de alcohol pudo incrementar el riesgo de CG (OR de 1,39). Tramacere y colaboradores ${ }^{(17)}$ encontraron una asociación entre CG y 
el consumo de alcohol solamente en los bebedores pesados ( $>4$ copas $x$ día). Otros estudios mostraron que las personas que consumen alcohol ( $>50 \mathrm{~g}$ al día) tuvieron un $24 \%$ más de riesgo para $\mathrm{CG}$ en comparación con otros que no consumen o consumen menos ${ }^{(8,9)}$.

Nemati y colaboradores ${ }^{(18)}$ reportaron que la baja ingesta de frutas y verduras fue un factor de riesgo. En contraste, la ingesta alta de frutas y verduras es un factor protector $(9$ estudios). El papel protector del consumo de frutas y verduras también fue observado en un estudio holandés con el consumo de $156 \mathrm{~g}$ x día ${ }^{(7,8)}$. Por otro lado, en un estudio de cohorte con personas vegetarianas se encontró que el riesgo fue bajo comparado con quienes ingieren carne ${ }^{(8)}$. El consumo excesivo de carnes rojas, comida ahumada, carnes procesadas y carnes saladas también fue reportado como factor de riesgo para CG en 8 estudios ${ }^{(8)}$.

La IARC ha clasificado el consumo de sal como uno de los factores de riesgo más importantes para el CG debido a los hallazgos reportados por múltiples estudios ${ }^{(13)}$. Se estima que el $24 \%$ de los casos en el Reino Unido estaban asociados con la ingesta de más de $6 \mathrm{~g}$ de sal al día. Los resultados del metaanálisis de D'Elia y colaboradores ${ }^{(19)}$ revelan que el riesgo de CG fue mayor en personas con ingesta alta de sal que en aquellos con ingesta baja. En la revisión sistemática publicada por $\mathrm{Ge}$ y colaboradores ${ }^{(20)}$ se reporta que la ingesta de sal incrementa el riesgo de CG en un $22 \%^{(8,9)}$.

Gómez y colaboradores ${ }^{(21)}$, en un trabajo observacional analítico prospectivo, compararon el consumo de alimentos y hábitos relacionados en dos grupos: uno con CG y otro con úlcera duodenal. Los autores encontraron asociaciones significativas entre los patrones de ingesta dietaria y el CG, por ejemplo, la alta ingesta de sal y el consumo de alimentos ahumados. Si bien el HP es un factor de riesgo establecido, también es claro que no es causa suficiente para el desarrollo del CG; en este sentido, la ingesta de sal puede tener un papel sinérgico con la infección en la causalidad de este tumor. También se encontró que el cáncer familiar, como factor de riesgo para CG, tuvo un OR global de 4,2, lo que coincide con otros estudios.

La identificación de los factores de riesgo puede proporcionar un entendimiento en la etiología de la enfermedad y puede sugerir estrategias de prevención. En este metaanálisis latinoamericano se identificó que el incremento en el riesgo para CG estuvo asociado con el tabaquismo, el consumo de alcohol, el alto consumo de carnes rojas y carnes procesadas, ingesta alta de sal y la disminución en el riesgo con niveles de educación más altos y con alto consumo de frutas y verduras ${ }^{(22)}$.

Los mismos trabajos de Yusefi y colaboradores ${ }^{(8)}$ y de Karimi y colaboradores ${ }^{(13)}$ muestran evidencia del efecto de los antecedentes familiares y la herencia en la incidencia de CG. Ellos encuentran que tener una historia familiar de CG es

un factor de riesgo para desarrollar CG. Asimismo, Yaghoobi y colaboradores ${ }^{(23)}$ reportaron que el riesgo de CG fue 2 a 10 veces mayor en personas con historia familiar de CG.

Los factores socioeconómicos se reportaron como relevantes en 10 estudios. Es conocida la mayor prevalencia de infección por HP en estratos socioeconómicos más bajos, poblaciones o grupos humanos con bajo nivel de instrucción y menor acceso a instalaciones sanitarias básicas, así como en los países en vías de desarrollo ${ }^{(8,9,13)}$.

Nishimoto y colaboradores ${ }^{(24)}$, en un estudio en Irán, encontraron que la mayor incidencia de CG estuvo asociada con un ingreso económico anual bajo, menor gasto anual en comida, menor consumo de frutas y vegetales, y mayores cifras de desempleo.

En otro metaanálisis ${ }^{(12)}$ se exploró la asociación entre el CG y 14 factores de riesgo potencialmente modificables. Se incluyeron 231 estudios, con una población total de 33831063 pacientes, y se expresó el riesgo en valores de OR. Los factores asociados de manera significativa con el riesgo de CG se recogen en la Tabla 1.

Tabla 1. Factores de riesgo para CG

\section{Factor de riesgo}

Helicobacter pylori

Tabaquismo

Antecedente de tabaquismo

Alcohol

Antecedente de ingesta de alcohol

Comida salada

Sal

Modificado de: Poorolajal J, et al. Epidemiology and Health. 2020;42:e2020004.

En resumen, se encuentra que la infección por HP, el tabaquismo, la alta ingesta de alcohol y de sal incrementan de forma significativa el riesgo de $\mathrm{CG}^{(7,12)}$.

La procedencia de zonas geográficas de alta incidencia de CG, la anemia perniciosa, el antecedente de resección gástrica parcial, la infección por el virus de Epstein-Barr (VEB), el tipo de sangre (tipo A), exposiciones ocupacionales a sustancias tóxicas como agroquímicos de fumigación, el trabajo con cemento, la fabricación de caucho, y la minería de carbón y con cromio también han sido mencionados como factores de riesgo. Estos últimos en relación con la dosis, duración y tiempo de exposición ${ }^{(8,9,14)}$.

Retomando varios de los conceptos mencionados, podemos decir que el CG es una enfermedad devastadora y poten- 
cialmente letal, con una historia natural larga y en la que se involucran múltiples factores, sin que hasta la fecha conozcamos una causa única ni última de este cáncer. Nuestra atención ha estado puesta ampliamente en la infección por HP como el factor de riesgo más importante, ya que puede estar relacionado con hasta el $90 \%$ de los casos; sin embargo, solo el $1 \%$ de los individuos infectados desarrollará CG, de modo que el HP es necesario, pero no suficiente ${ }^{(7)}$.

Todo esto nos lleva a pensar que otros factores de riesgo pueden ser tan importantes como la infección por HP, y muy probablemente ejercen su papel modulando el impacto tisular de la infección crónica en la mucosa gástrica ${ }^{(11)}$. En esa interacción entre el huésped y el hospedero, única y diferente para cada sujeto, que abre el camino hacia la gastritis crónica, la enfermedad ulcerosa péptica o hacia el cáncer, puede estar al menos una de las piezas que le faltan a nuestro rompecabezas.

\section{Modelos de predicción de riesgo}

Múltiples autores han desarrollado modelos de predicción de riesgo con base en los factores de riesgo. Buckland y colaboradores ${ }^{(25)}$ evaluaron una cohorte para medir el impacto de los factores asociados con el estilo de vida en la ocurrencia de CG; por su parte, Tata y colaboradores $^{(26)}$ desarrollaron un sistema diagnóstico con un puntaje basado en variables clínicas.

Sin embargo, estos modelos no involucran la presencia o ausencia de la infección por HP ni la presencia de lesiones o condiciones premalignas como la gastritis atrófica $(\mathrm{GA})^{(11)}$. Por el contrario, otros trabajos dejan de lado los aspectos clínicos y de estilo de vida para centrarse únicamente en la evaluación de los dos primeros factores mencionados y combinan la detección de anticuerpos contra HP y los niveles séricos de pepsinógeno I y II, que pueden considerarse como la expresión bioquímica del fenómeno histológico que es la GA. Ambos tipos de pepsinógeno se producen en la mucosa del fondo y del cuerpo gástrico y el tipo II, fundamentalmente en el antro; a medida que la atrofia gástrica progresa de su territorio inicial (que suele ser el antro) y compromete progresivamente el cuerpo, la producción de pepsinógeno II aumenta mientras que la del tipo I disminuye, y la relación I/II se reduce. En ese mismo sentido, la mucosa atrófica produce menos ácido y la gastrina sérica se eleva ${ }^{(27)}$.

Cuando se utiliza la medición de los niveles de anticuerpos contra HP y los niveles séricos de pepsinógeno I y II, se involucran solo dos factores de riesgo. Como el CG es multifactorial, creemos que la herramienta de predicción de riesgo también debe serlo.

Iida y colaboradores ${ }^{(28)}$ llevaron a cabo un estudio en la ciudad de Hisayama (Japón) con el objetivo de desarrollar y evaluar una herramienta de medición del riesgo individual de CG. Residentes mayores de 40 años asintomáticos fueron elegidos y seguidos desde 1988 a 2002 como la primera cohorte de estudio, que tuvo 2444 individuos, con un seguimiento de 14 años, y desde 2002 a 2007 la cohorte de validación, que tuvo 3204 sujetos, con 5 años de seguimiento. Durante el seguimiento se encontraron 90 casos de CG en la cohorte de estudio y 35 casos en la cohorte de validación. Con la cohorte de estudio elaboraron el modelo de predicción del riesgo utilizando factores de riesgo significativos; posteriormente, esta herramienta fue validada internamente. En el análisis univariado, la edad, el género, la combinación de los anticuerpos contra HP y los valores séricos de pepsinógeno I y II, el nivel de la hemoglobina glucosilada $\left(\mathrm{HbA}_{\mathrm{lc}}\right)$, el tabaquismo y la ingesta de alcohol estuvieron significativamente asociados con la incidencia de CG. En el análisis multivariado, con excepción del consumo de alcohol, todos los factores seguían estando significativamente asociados con la incidencia de CG. Se asignó un puntaje a cada factor de riesgo. El peso de cada factor (puntaje asignado) para predecir el riesgo de CG se determinó con la herramienta de evaluación en función de los coeficientes de un modelo de riesgos proporcionales de Cox en la cohorte de estudio.

En este trabajo, el total del puntaje fue calculado como la suma de los puntajes de riesgo para los diferentes factores. El punto de corte para la predicción del riesgo de CG fue de 8 . En los sujetos con puntaje de 8 o más, el riesgo para desarrollo de CG se incrementó en 5,3 veces (IC del $95 \%$ 3,4 a 8,2) con respecto a quienes tuvieron un puntaje de 7 o menos. La recomendación fue estudiar con endoscopia digestiva alta (EDA) a quienes tenían 8 o más puntos (Tabla 2).

Eom y colaboradores ${ }^{(29)}$ también desarrollaron un modelo de predicción utilizando una cohorte de seguimiento y otra de validación. Dentro del modelo se incluyeron: edad, índice de masa corporal (IMC), historia familiar de cáncer, ingesta de sal, consumo de alcohol y tabaquismo. Este modelo de predicción mostró buena precisión tanto en la cohorte de estudio como en la de validación. En este trabajo se propuso que la población general con factores de riesgo se derive a un chequeo médico regular. La infección por HP no fue tenida en cuenta.

Varios trabajos realizados en Asia y, particularmente, en China mencionan la población de alto riesgo para CG como sujetos que viven en áreas de alta incidencia por más de tres años, con una historia familiar de CG y factores de riesgo como ingesta alta de sal, tabaquismo o ingesta pesada de alcohol. Por tener una población tan grande, la EDA no es fácil de aplicar masivamente y genera altos costos; por esta razón, han establecido una herramienta de estratificación como tamizaje previo a la endoscopia, que se aplica predominantemente en esos grupos poblacionales considerados globalmente de alto riesgo para identificar a los individuos con mayor riesgo real de desarrollar $\mathrm{CG}^{(30)}$. 
Tabla 2. Modelos de predicción de riesgo para CG

\begin{tabular}{|c|c|c|c|c|c|}
\hline \multicolumn{2}{|c|}{ Factor de riesgo } & \multicolumn{4}{|c|}{ Autores } \\
\hline Variable & Rango & $\begin{array}{c}\text { lida } \\
\text { Puntaje }\end{array}$ & $\begin{array}{c}\text { Cai } \\
\text { Puntaje }\end{array}$ & $\begin{array}{l}\text { Chavart } \\
\text { Puntaje } \\
\text { (Mujer) }\end{array}$ & $\begin{array}{l}\text { Chavart } \\
\text { Puntaje } \\
\text { (Hombre) }\end{array}$ \\
\hline \multirow{7}{*}{$\begin{array}{l}\text { Edad } \\
\text { (años) }\end{array}$} & $40-44$ & 0 & 0 & 0 & 1 \\
\hline & $45-49$ & 0 & 0 & 1 & 3 \\
\hline & $50-54$ & 2 & 4 & 2 & 4 \\
\hline & $55-59$ & 2 & 4 & 3 & 6 \\
\hline & $60-64$ & 3 & 6 & 4 & 8 \\
\hline & $65-69$ & 3 & 6 & 5 & 10 \\
\hline & $>70$ & 2 & 9 & & \\
\hline \multirow[t]{2}{*}{ Sexo } & Hombre & 3 & 4 & & \\
\hline & Mujer & 0 & 0 & & \\
\hline HP & HP (-) GA (-) & 0 & 0 & 0 & 0 \\
\hline \multirow[t]{3}{*}{ GA } & $\mathrm{HP}(+) \mathrm{GA}(-)$ & 2 & 1 & 8 & 8 \\
\hline & $\mathrm{HP}(+) \mathrm{GA}(+)$ & 5 & 3 & 11 & 11 \\
\hline & $\mathrm{HP}(-) \mathrm{GA}(+)$ & 5 & 3 & 11 & 11 \\
\hline \multirow{2}{*}{$\begin{array}{l}\text { Taba- } \\
\text { quismo }\end{array}$} & Sí & 1 & & & 1 \\
\hline & No & & & & 0 \\
\hline \multirow[t]{2}{*}{ Sal } & Sí & & 2 & 1 & 1 \\
\hline & No & & 0 & 0 & 0 \\
\hline Puntaje & & > a 8 & $\begin{array}{c}\text { 0-11: bajo } \\
\text { 12-16: medio } \\
\text { 17-25: alto }\end{array}$ & > a 20 & > a 20 \\
\hline
\end{tabular}

Modificado de: Charvat H, et al. Int J Cancer. 2016;138(2):320-31; Iida M, et al. Gastric Cancer. 2018;21(3):383-90; Cai Q et al. Gut. 2019;68:1576-87.

Cai y colaboradores ${ }^{(30)}$ publicaron un trabajo multicéntrico de 115 hospitales (desde junio de 2015 hasta marzo de 2017) con una población de alto riesgo (incidencia de más de 30 por cada 100000 habitantes) por factores como edad, género, historia familiar de cáncer, dieta con alta ingesta de sal, GA, infección por HP, procedencia geográfica, tabaquismo e ingesta de alcohol. Utilizando una cohorte de estudio y otra de validación, desarrollaron una herramienta para predecir el riesgo de CG. Un cuestionario registró los siguientes datos: edad, género, peso corporal, IMC, tabaquismo definido como más de un cigarrillo al día por un año (si la respuesta era sí, el número de cigarrillos y la duración se registraron), consumo de alcohol (cualquier tipo de alcohol una vez por semana durante el último año; si la respuesta es sí, clase de alcohol y frecuencia de consumo), hábitos dietarios (consumo de sal más de $10 \mathrm{~g} /$ día), consumo de encurtidos, comida frita, ahumados, carne roja, vegetales verdes, frutas frescas (consumo frecuente considerado 3 o más por semana) e historia familiar de CG.

En todos los pacientes se determinó el nivel sérico de pepsinógeno I y II, gastrina y anticuerpos del tipo inmunoglobulina $\mathrm{G}(\mathrm{IgG})$ contra HP. Finalmente, los pacientes escogidos fueron llevados a una endoscopia con luz blanca y biopsias del antro, incisura y cuerpo, y examen endoscópico con imágenes mejoradas tipo NBI (Narrow Band Imaging).

Los cuatro procedimientos: cuestionario, laboratorios, gastroscopia y patología fueron elaborados por los investigadores. Dos tercios de los participantes fueron aleatorizados a la cohorte de estudio y un tercio a la cohorte de validación.

En la cohorte de estudio (9838 participantes) se encontraron 267 sujetos con CG $(2,7 \%)$ y en la de validación (5091 participantes) 138 (2,7\%). En el análisis univariado, se encontraron 17 variables como potencialmente asociadas con CG $(p<0,25)$. Las que presentaron más poder estadístico fueron edad, género, relación pepsinógeno I/ II, gastrina y anticuerpos contra HP, consumo de comida salada y comidas fritas (todas con un valor de $p<0,05$ ).

Los puntajes variaban entre 0 a 25 . Se establecieron grupos de riesgo en las categorías baja, media y alta cuando los puntajes variaban entre 0 a 11,12 a 16 y de 17 a 25 , respectivamente. El 66,7 \% fueron considerados de bajo riesgo, el $27,6 \%$ de riesgo medio y el 5,7 \% de riesgo alto. La prevalencia de CG fue de $1,2 \%, 4,4 \%$ y $12,3 \%$, respectivamente $(p<0,001)$ (Tabla 2).

El 70,8\% de los pacientes con cáncer gástrico avanzado (CGA) y el 70,3\% de los pacientes con cáncer gástrico temprano (CGT) fueron diagnosticados cuando la endoscopia se realizó en los grupos considerados de riesgo medio $y$ alto por el puntaje previamente mencionado.

Este grupo de factores (edad, género, relación pepsinógeno I/II, gastrina, estado serológico frente a HP, consumo de comida salada y comida frita) es interesante, porque todos han sido identificados como predictores independientes de riesgo para CG y los resultados son consistentes con otros estudios.

Otro trabajo de Chavart y colaboradores ${ }^{(11)}$ desarrollaron un modelo de predicción para estimar la probabilidad de ocurrencia de CG a 10 años con base en una cohorte de 19 028 individuos. Para esto, combinaron variables demográficas y clínicas (edad, género, tabaquismo, consumo de sal e historia familiar) con el estado de anticuerpos IgG contra HP y niveles séricos de pepsinógeno I y II.

En concordancia con estudios previos, los autores reportaron que la infección por HP y la GA fueron factores de riesgo significativos para la ocurrencia del CG. En este estudio no se encontró una diferencia en el riesgo entre las categorías $\mathrm{C}$ 
y D (del método $\mathrm{ABC}$ cuando se relaciona la serología para HP con los niveles de pepsinógeno I y II). También encontraron un peso importante para la edad, especialmente en hombres, el tabaquismo y la ingesta de sal como predictores independientes de la ocurrencia del CG. La probabilidad de CG a los 10 años fue de $0,04 \%$ a 14,87 \% para los hombres y de $0,03 \%$ a $4,91 \%$ para mujeres ${ }^{(11)}$ (Tabla 2).

La edad tuvo un efecto importante en los hombres, lo cual incrementó el riesgo más de 5 veces a los 60 años y más de 10 veces por encima de 70 años en comparación con los hombres a los 40 años $(1,58 \% \text { y } 3,56 \% \text { frente a } 0,31 \%)^{(11)}$.

El puntaje variaba de 0 a 24 . Individuos con puntaje de 10 o menos tenían una probabilidad acumulada de CG a los 10 años de menos del $0,4 \%$, mientras que esta probabilidad es $>5 \%$ para individuos con puntaje de $20 \mathrm{o}>20$.

Todos estos estudios ${ }^{(11,28,30)}$ abordan el problema de la predicción del riesgo de CG utilizando herramientas que involucran factores clínicos, de estilo de vida y serológicos (que pueden expresar la funcionalidad de la mucosa gástrica), con el fin de identificar los individuos que pueden beneficiarse más de la EDA.

Se realizó un metaanálisis ${ }^{(27)}$ para evaluar la predicción de desarrollo de CG por medición de los niveles séricos de pepsinógenos, prueba de anticuerpos contra HP y un modelo de predicción del riesgo basado en estas dos pruebas. Este modelo está categorizado en 4 grupos: bajo riesgo A (Ac HP - y PG -), moderado riesgo B (Ac HP + y PG -) y alto riesgo $\mathrm{C}(\mathrm{Ac} \mathrm{HP}+\mathrm{y} \mathrm{PG}+)$ y $\mathrm{D}(\mathrm{Ac} \mathrm{HP}-\mathrm{y} \mathrm{PG}+)$. Este estudio incluyó 9 cohortes prospectivas de países de Oriente con un total de 33741 participantes asintomáticos en programas de tamizaje para CG. La edad media de los participantes varió de 45 a 57 años, mientras que el seguimiento estuvo entre 3,9 a 14 años. Este estudio encontró que los adultos con PG + tuvieron aproximadamente 4 veces más riesgo de desarrollar CG que aquellos con pruebas negativas ${ }^{(27,31)}$.

Como hemos mencionado, el nivel sérico bajo del pepsinógeno I y la relación del pepsinógeno I/II reflejan la gravedad de la atrofia gástrica. Estos datos en combinación con la presencia de anticuerpos contra HP y la gastrina han sido utilizados para identificar a individuos con alto riesgo para $\mathrm{CG}^{(28)}$.

Las guías japonesas de diagnóstico temprano de CG recomiendan el uso de la endoscopia o la serie gastrointestinal superior con medio de contraste para el tamizaje de oportunidad, enfatizando que la primera es más sensible que la segunda. En la actualidad, los estudios serológicos de pepsinógeno y de anticuerpos no se recomiendan como tamizaje en esta misma guía debido a la falta de evidencia en la reducción de la mortalidad del $\mathrm{CG}^{(32)}$.

En la guía MAPS se recomienda que la endoscopia debe ser considerada en individuos por encima de los 50 años con múltiples factores de riesgo (hombres, fumadores, anemia perniciosa), y particularmente para aquellos con familiares en primer grado con $\mathrm{CG}^{(10)}$. En esta misma guía los autores consideran que no hay suficiente evidencia que soporte el uso de los estudios serológicos de pepsinógeno $y$ de anticuerpos contra HP en los programas de tamizaje, y menos aún en áreas de baja incidencia de $\mathrm{CG}^{(10)}$.

\section{APORTE ENDOSCÓPICO}

Comparado con otros factores de riesgo, la GA y la metaplasia intestinal (MI) incrementan el riesgo de CG de forma exponencial. Por esta razón, se pueden considerar como de alto riesgo a los individuos con estos hallazgos ${ }^{(7)}$.

\section{Gastritis crónica atrófica}

Es bien conocida la hipótesis de que el CG se desarrolla a través de una cascada de lesiones precursoras después de la infección por HP. Un estudio holandés ${ }^{(33)}$ mostró que el riesgo de CG se incrementa con cada paso de esta cascada de acuerdo con la gravedad de las lesiones premalignas gástricas. La incidencia anual de CG a los 5 años después del diagnóstico: 0,1\% para GA, 0,25\% para MI, 0,6 \% para displasia moderada y de $6 \%$ para displasia grave. Por su parte, el riesgo de CG en individuos con GA varía de acuerdo con la gravedad de la misma; además, se reportó una alta razón de tasa ajustadas (adjusted rate ratio $[\mathrm{RR}]$ ) de CG de los pacientes con atrofia grave corporal, de 5,76, en comparación con pacientes que tuvieran poca atrofia o no la tuvieran ${ }^{(34)}$.

\section{Metaplasia intestinal}

En el estudio holandés, la incidencia anual de CG a los 5 años del diagnóstico de $\mathrm{MI}$ es de $0,25 \%$. Otro estudio epidemiológico ${ }^{(35)}$ sugiere que los pacientes con MI tienen 10 veces más riesgo de desarrollar CG. En un estudio chino ${ }^{(36)}$ realizado en una zona de alto riesgo de CG los residentes con lesiones precancerosas fueron seguidos por 5 años, y el OR para CG en los sujetos con MI fue de 17,1 a 29,3.

El riesgo de CG también depende de la extensión y el fenotipo de la MI. Se habla de MI completa cuando la mucosa del estómago se parece histológicamente a la mucosa del intestino delgado. Por otra parte, se habla de MI incompleta cuando el epitelio de la mucosa gástrica se parece a la mucosa colónica. La MI puede clasificarse como tipo I, II y III de acuerdo con el fenotipo de la mucina. La MI tipo I completa (solo expresa sialomucinas) y tipo III incompleta (expresa sulfomucinas). La MI tipo II incompleta es un híbrido que expresa una mezcla de mucinas gástricas e intestinales. Varios reportes hablan de que el riesgo de CG 
es mayor en la MI tipo III; sin embargo, otros estudios han dicho lo contrario. Parece ser que el fenotipo de la MI no es determinante en la predicción o desarrollo del CG; no obstante, otros estudios han mostrado que la prevalencia de MI incompleta fue significativamente mayor en pacientes con CG que en otras lesiones gástricas. Además, está reportado que más de la mitad de los estudios encuentran una relación estadística entre la MI y el desarrollo subsecuente de CG (riesgo relativo para CG de 4 a 11 veces mayor con la presencia de $\mathrm{MI}$ incompleta en comparación a la completa). Los autores concluyen que la subclasificación del fenotipo es de utilidad como predictor de riesgo de $\mathrm{CG}^{(7)}$.

La MI tiende a aparecer primero en la incisura angularis y se extiende a la mucosa vecina en ambos sentidos hacia el antro y el cuerpo. Un estudio que revisa los modelos de distribución de la MI muestra que la extensión de la misma está significativamente asociada con un incremento en el riesgo de CG. En conclusión, se ha propuesto que la distribución de la MI, más que el subtipo, puede ser de mayor valor predictivo de riesgo para $\mathrm{CG}^{(7)}$.

\section{GRUPOS DE ALTO RIESGO}

Los factores de riesgo identificados para CG difieren en su OR. Comparados con otros factores, la GA y la MI incrementan marcadamente el riesgo de CG. Una pregunta clave en el manejo de estos pacientes con alto riesgo es cómo seleccionar un grupo de mayor riesgo entre los sujetos con GA y $\mathrm{MI}^{(7)}$.

Existen varias formas de identificar a individuos con alto riesgo para el desarrollo de CG, como los métodos no invasivos (pepsinógeno y anticuerpos contra HP), la endoscopia y la histología. Un examen histológico es necesario para el diagnóstico de una lesión gástrica precancerosa. La endoscopia, especialmente con tecnología moderna (imágenes mejoradas), tiene una precisión aceptable en el diagnóstico de estas lesiones. Actualmente, el principal abordaje en países occidentales es la endoscopia con histología, mientras que en los países orientales con alta prevalencia de CG se utiliza solo la endoscopia ${ }^{(31)}$.

La medición de riesgo preendoscópico basado en las características clínicas y demográficas tales como edad, etnia, género, tabaquismo e infección por HP es útil para identificar individuos con alta probabilidad preprueba para un posible abordaje costoefectivo, especialmente en países con riesgo bajo e intermedio ${ }^{(31)}$.

La estratificación histológica del riesgo de CG implica medir la extensión y gravedad de la GA y la $\mathrm{MI}^{(4)}$. En países asiáticos, la extensión de la atrofia con frecuencia se mide endoscópicamente a través de la observación de la mucosa, utilizando la clasificación de Kimura-Takemoto, así como la presencia de metaplasia y su localización. En Occidente se prefiere la clasificación histológica realizada en las biopsias sistemáticas que incluyen cuerpo y antro, y que son informadas por el patólogo usando el sistema OLGA (Operative Link on Gastritis Assessment) u OLGIM (Operative Link on Gastric Intestinal Metaplasia Assessment) ${ }^{(5)}$. Ambos abordajes requieren la endoscopia realizada en un estómago limpio y con el tiempo de observación suficiente. En un caso, se requieren biopsias de sitios específicos; en el otro, entrenamiento en la clasificación endoscópica ${ }^{(4)}$.

OLGA está basado en una combinación del puntaje de la atrofia con muestras obtenidas de la mucosa antral y oxíntica (protocolo de Sídney). Los valores del puntaje combinados expresan el "estado de la mucosa" y representan un mensaje de la gravedad de la atrofia, la cual es paralela al riesgo de CG (OLGA estados O, I y II: bajo riesgo, OLGA III y IV: alto riesgo de CG). Estudios prospectivos han respaldado el valor pronóstico del estadiaje de la GA y su utilidad en la práctica clínica ${ }^{(2,37)}$.

El mecanismo exacto, la contribución de los factores de riesgo medio ambientales y la susceptibilidad genética del huésped participan en la progresión de la carcinogénesis gástrica y no han sido todavía completamente aclarados. El $20 \%$ de los pacientes con CG pueden tener una historia familiar de CG. El riesgo es 2 veces mayor en los hombres que en las mujeres y usualmente se presenta entre los $60 \mathrm{a}$ 80 años. El $18 \%$ de los CG son atribuidos al tabaquismo y cuando el individuo además de fumar ingiere alcohol, el riesgo de CG se incrementa hasta 5 veces ${ }^{(38)}$.

No más del $30 \%$ de los pacientes con CG sobreviven más de 5 años después del diagnóstico, debido a que la mayoría se diagnostican en estadios avanzados ${ }^{(5)}$. Sin embargo, muchos pacientes consultan en algún momento previo a la aparición del CG; la mayoría incluso llega a un estudio endoscópico, pero raramente se enfocan teniendo en cuenta los factores de riesgo asociados con su historia particular, los cuales, sumados a una muy buena información endoscópica e histopatológica, nos permitirían no solo definir su seguimiento sino precisar programas de prevención en relación con su enfermedad.

La incorporación de factores de riesgo dentro de modelos de predicción podría permitir una selección más adecuada de pacientes con riesgo de CG para tamizaje. Un gran número de modelos de predicción han sido desarrollados, sin embargo, ninguno de ellos es perfecto; cada uno tiene sus limitaciones y requieren de validación en grandes poblaciones antes de que su implementación clínica pueda recomendarse ${ }^{(39)}$.

En regiones geográficas con alta carga de la enfermedad para CG estas herramientas de trabajo, que incluyen evaluación de factores de riesgo e información endoscópica, pueden tener cabida como programas para seguimiento $y$ prevención ${ }^{(39)}$. 
La detección de lesiones gástricas premalignas generalmente requiere endoscopia más biopsias. Sin embargo, existen características demográficas y clínicas que son útiles para predecir la presencia de estas lesiones incluyendo lugar de origen, etnia, género, edad, historia familiar de CG, infección por HP y niveles séricos de pepsinógeno ${ }^{(31)}$.

Un estudio encontró que los individuos que inmigraron a los Estados Unidos desde áreas de alto riesgo (chinos y latinoamericanos) tienen un mayor riesgo de desarrollar CG en comparación con los nativos, al igual que los individuos infectados con HP y con familiares en primer grado con CG. El género masculino, el tabaquismo y la edad avanzada están también asociados con un mayor riesgo de desarrollo de $\mathrm{CG}^{(31)}$.

La medición del riesgo antes de la endoscopia es posible a través de un entendimiento del papel de los factores de riesgo y es útil para seleccionar individuos con alta probabilidad preprueba, especialmente en regiones con riesgo bajo e intermedio ${ }^{(11)}$.

El CG es un problema de salud pública con más de un millón de casos nuevos diagnosticados cada año en el mundo. A pesar de la declinación en su incidencia y mortalidad en los últimos 5 años, el CG permanece como la tercera causa de muerte relacionada por cáncer en el mundo ${ }^{(38)}$. La modificación de la dieta y los estilos de vida es la forma más racional de prevención del CG. El consumo de frutas, evitar el cigarrillo y la ingesta de alcohol, mantener un peso corporal adecuado, la actividad física y la no ingesta excesiva de sal ni comidas ahumadas ayudan a disminuir el riesgo de esta enfermedad ${ }^{(14)}$.

\section{CONCLUSIÓN}

Las estrategias de prevención primaria y secundaria tales como las modificaciones dietarias y los programas de tamizaje son medidas muy importantes para reducir el riesgo de CG. Sin embargo, los factores de riesgo no son tenidos en cuenta al momento de establecer las estrategias de seguimiento. Estos factores, al igual que la infección por $\mathrm{HP}$, pueden estar actuando durante muchos años o incluso décadas y solamente nos preocupamos por erradicar el HP. Es probable que el desarrollo del CG se derive de la interrelación de todos estos factores, tanto los modificables como los no modificables, y si les damos contexto dentro del aporte clínico podríamos impactar más la enfermedad.

Por ello, evaluar el peso real de los factores de riesgo ayudaría a definir grupos de alto y bajo riesgo, a determinar la pertinencia del procedimiento endoscópico y su frecuencia, lo mismo que las diferentes estrategias de prevención.

La identificación de los factores de riesgo puede proporcionar un entendimiento en la etiología de la enfermedad y sugerir estrategias de prevención. El conocimiento de la epidemiología, la historia natural y los factores de riesgo debe ser esencial en la práctica del gastroenterólogo y del cirujano para personalizar las decisiones acerca de la estratificación del riesgo, el tamizaje y la prevención.

Creemos que es necesario desarrollar una herramienta de estudio basada en la identificación de factores de riesgo de cada individuo, que se pueda sumar a los hallazgos endoscópicos e histológicos y ser usada en la práctica clínica para la clasificación del riesgo de $\mathrm{CG}^{(11)}$ (Figura 2).

Cada día encontramos más literatura sobre quimioterapia a la medida de cada tumor, pero poco en relación con estrategias de prevención y seguimiento a la medida de cada paciente o de cada grupo poblacional. Nos gustaría propender por una cultura médica de evaluación del riesgo a la medida de manera que indaguemos activamente por todos los factores de riesgo mencionados previamente, $y$ los tengamos en cuenta de manera activa y permanente en las decisiones clínicas.

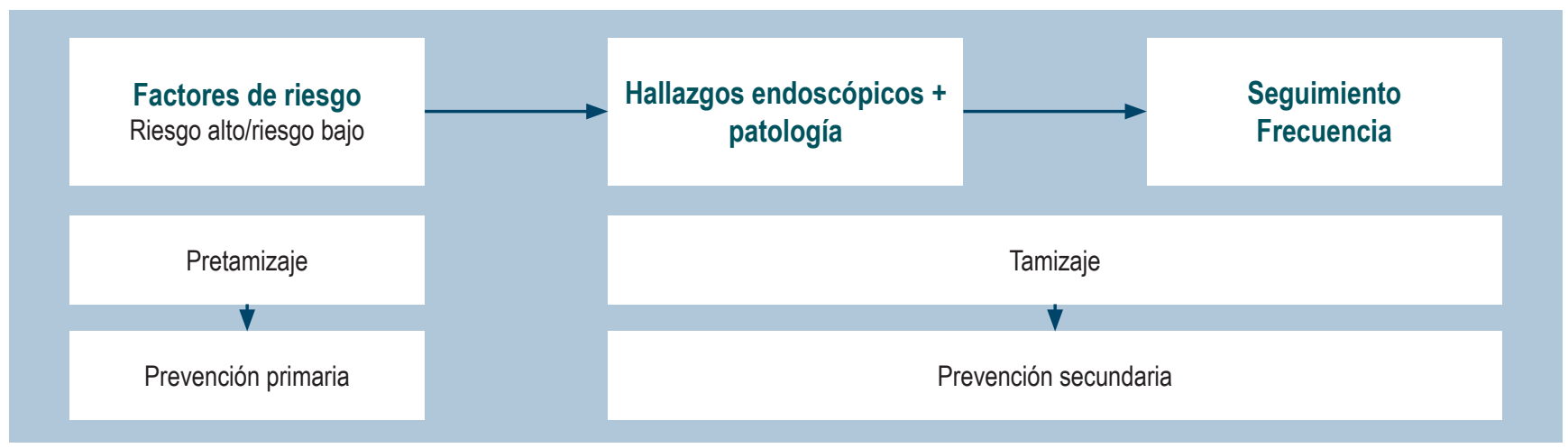

Figura 2. Utilidad de factores de riesgo. Elaboración propia. 


\section{REFERENCIAS}

1. Caicedo HH, Hashimoto DA, Caicedo JC, Pentland A, Pisano GP. Overcoming barriers to early disease intervention. Nat Biotechnol. 2020;38(6):669-73. https://doi. org/10.1038/s41587-020-0550-z

2. Graham DY, Rugge M, Genta RM. Diagnosis: gastric intestinal metaplasia - what to do next? Curr Opin Gastroenterol. 2019;35(6):535-43. https://doi.org/10.1097/MOG.0000000000000576

3. Chadwick G, Groene O, Riley S, Hardwick R, Crosby T, Hoare J, Hanna GB, Greenaway K, Cromwell DA. Gastric Cancers Missed During Endoscopy in England. Clin Gastroenterol Hepatol. 2015;13(7):1264-1270.e1.https:// doi.org/10.1016/j.cgh.2015.01.025

4. Rugge M, Capelle LG, Fassan M. Individual risk stratification of gastric cancer: evolving concepts and their impact on clinical practice. Best Pract Res Clin Gastroenterol. 2014;28(6):1043-53. https://doi.org/10.1016/j. bpg.2014.09.002

5. Rugge M, Sugano K, Scarpignato C, Sacchi D, Oblitas WJ, Naccarato AG. Gastric cancer prevention targeted on risk assessment: Gastritis OLGA staging. Helicobacter. 2019;24(2):e12571. https://doi.org/10.1111/hel.12571

6. van der Kaaij RT, van Kessel JP, van Dieren JM, Snaebjornsson $\mathrm{P}$, Balagué $\mathrm{O}$, van Coevorden F, van der Kolk LE, Sikorska K, Cats A, van Sandick JW. Outcomes after prophylactic gastrectomy for hereditary diffuse gastric cancer. Br J Surg. 2018;105(2):e176-e182. https://doi.org/10.1002/bjs.10754

7. Yoon $\mathrm{H}, \mathrm{Kim} \mathrm{N}$. Diagnosis and management of high risk group for Gastric Cancer. Gut and Liver 2015;9(1): 5-17. https://doi.org/10.5009/gnl14118

8. Yusefi AR, Bagheri Lankarani K, Bastani P, Radinmanesh M, Kavosi Z. Risk Factors for Gastric Cancer: A Systematic Review. Asian Pac J Cancer Prev. 2018;19(3):591-603. https://doi.org/10.22034/APJCP.2018.19.3.591

9. Lyons K, Le LC, Pham YT, Borron C, Park JY, Tran CTD, Tran TV, Tran HT, Vu KT, Do CD, Pelucchi C, La Vecchia C, Zgibor J, Boffetta P, Luu HN. Gastric cancer: epidemiology, biology, and prevention: a mini review. Eur J Cancer Prev. 2019;28(5):397-412. https://doi.org/10.1097/ CEJ.0000000000000480

10. Banks M, Graham D, Jansen M, Gotoda T, Coda S, di Pietro M, Uedo N, Bhandari P, Pritchard DM, Kuipers EJ, Rodriguez-Justo M, Novelli MR, Ragunath K, Shepherd N, Dinis-Ribeiro M. British Society of Gastroenterology guidelines on the diagnosis and management of patients at risk of gastric adenocarcinoma. Gut. 2019;68(9):1545-75. https://doi.org/10.1136/gutjnl-2018-318126

11. Charvat $H$, Sasazuki S, Inoue M, Iwasaki M, Sawada N, Shimazu T, Yamaji T, Tsugane S; JPHC Study Group. Prediction of the 10-year probability of gastric cancer occurrence in the Japanese population: the JPHC study cohort II. Int J Cancer. 2016;138(2):320-31. https://doi. org/10.1002/ijc.29705
12. Poorolajal J, Moradi L, Mohammadi Y, Cheraghi Z, Gohari-Ensaf F. Risk factors for stomach cancer: a systematic review and meta-analysis. Epidemiol Health. 2020;42:e2020004. https://doi.org/10.4178/epih. e2020004

13. Karimi P, Islami F, Anandasabapathy S, Freedman ND, Kamangar F. Gastric cancer: descriptive epidemiology, risk factors, screening, and prevention. Cancer Epidemiol Biomarkers Prev. 2014;23(5):700-13. https://doi. org/10.1158/1055-9965.EPI-13-1057

14. Rawla P, Barsouk A. Epidemiology of gastric cancer: global trends, risk factors and prevention. Prz Gastroenterol. 2019;14(1):26-38. https://doi.org/10.5114/ pg.2018.80001

15. Huang JQ, Sridhar S, Chen Y, Hunt RH. Meta-analysis of the relationship between Helicobacter pylori seropositivity and gastric cancer. Gastroenterology. 1998;114(6):116979. https://doi.org/10.1016/S0016-5085(98)70422-6

16. Ma K, Baloch Z, He TT, Xia X. Alcohol Consumption and Gastric Cancer Risk: A Meta-Analysis. Med Sci Monit. 2017;23:238-46. https://doi.org/10.12659/MSM.899423

17. Tramacere I, Negri E, Pelucchi C, Bagnardi V, Rota M, Scotti L, Islami F, Corrao G, La Vecchia C, Boffetta P. A meta-analysis on alcohol drinking and gastric cancer risk. Ann Oncol. 2012;23(1):28-36. https://doi.org/10.1093/ annonc/mdr135

18. Nemati A, Mahdavi R, Naghizadeh Baghi A. Case-control study of dietary pattern and other risk factors for gastric cancer. Health Promot Perspect. 2012;2(1):20-7. https:// doi.org/10.5681/hpp.2012.003

19. D’Elia L, Rossi G, Ippolito R, Cappuccio FP, Strazzullo P. Habitual salt intake and risk of gastric cancer: a meta-analysis of prospective studies. Clin Nutr. 2012;31(4):489-98. https://doi.org/10.1016/j.clnu.2012.01.003

20. Ge S, Feng X, Shen L, Wei Z, Zhu Q, Sun J. Association between Habitual Dietary Salt Intake and Risk of Gastric Cancer: A Systematic Review of Observational Studies. Gastroenterol Res Pract. 2012;2012:808120. https://doi. org/10.1155/2012/808120

21. Gómez-Zuleta M, Otero-Regino W, Ruiz-Lobo X. Factores de riesgo para cáncer gástrico en pacientes colombianos. Rev Colomb Gastroenterol. 2009;24(2):134-143.

22. Bonequi P, Meneses-González F, Correa P, Rabkin CS, Camargo MC. Risk factors for gastric cancer in Latin America: a meta-analysis. Cancer Causes Control. 2013;24(2):217-31. https://doi.org/10.1007/s10552012-0110-z

23. Yaghoobi M, Bijarchi R, Narod SA. Family history and the risk of gastric cancer. Br J Cancer. 2010;102(2):237-42. https://doi.org/10.1038/sj.bjc.6605380

24. Nishimoto IN, Hamada GS, Kowalski LP, Rodrigues JG, Iriya K, Sasazuki S, Hanaoka T, Tsugane S. Risks factors for 
stomach cáncer in Brazil(I): A case -control study among non Japanese Brazilians in Sao Paulo. Jpn J Clin Oncol. 2002;32(8):277-83. https://doi.org/10.1093/jjco/hyf060

25. Buckland G, Travier N, Huerta JM, Bueno-de-Mesquita HB, Siersema PD, Skeie G, Weiderpass E, Engeset D, Ericson U, Ohlsson B, Agudo A, Romieu I, Ferrari P, Freisling H, Colorado-Yohar S, Li K, Kaaks R, Pala V, Cross AJ, Riboli E, Trichopoulou A, Lagiou P, Bamia C, BoutronRuault MC, Fagherazzi G, Dartois L, May AM, Peeters PH, Panico S, Johansson M, Wallner B, Palli D, Key TJ, Khaw KT, Ardanaz E, Overvad K, Tjønneland A, Dorronsoro M, Sánchez MJ, Quirós JR, Naccarati A, Tumino R, Boeing $\mathrm{H}$, Gonzalez CA. Healthy lifestyle index and risk of gastric adenocarcinoma in the EPIC cohort study. Int J Cancer. 2015;137(3):598-606. https://doi.org/10.1002/ijc.29411

26. Tata MD, Gurunathan R, Palayan K. MARK >s Quadrant scoring system: a symptom-based targeted screening tool for gastric cancer. Ann Gastroenterol. 2014;27(1):34-41.

27. Chen XZ, Huang CZ, Hu WX, Liu Y, Yao XQ. Gastric Cancer Screening by Combined Determination of Serum Helicobacter pylori Antibody and Pepsinogen Concentrations: ABC Method for Gastric Cancer Screening. Chin Med J (Engl). 2018;131(10):1232-39. https://doi.org/10.4103/0366-6999.231512

28. Iida M, Ikeda F, Hata J, Hirakawa Y, Ohara T, Mukai N, Yoshida D, Yonemoto K, Esaki M, Kitazono T, Kiyohara Y, Ninomiya T. Development and validation of a risk assessment tool for gastric cancer in a general Japanese population. Gastric Cancer. 2018;21(3):383-90. https:// doi.org/10.1007/s10120-017-0768-8

29. Eom BW, Joo J, Kim S, Shin A, Yang HR, Park J, Choi IJ, Kim YW, Kim J, Nam BH. Prediction Model for Gastric Cancer Incidence in Korean Population. PLoS One. 2015;10(7):e0132613. https://doi.org/10.1371/journal. pone. 0132613

30. Cai Q, Zhu C, Yuan Y, Feng Q, Feng Y, Hao Y, Li J, Zhang K, Ye G, Ye L, Lv N, Zhang S, Liu C, Li M, Liu Q Li R, Pan J, Yang X, Zhu X, Li Y, Lao B, Ling A, Chen H, Li X, Xu P, Zhou J, Liu B, Du Z, Du Y, Li Z; Gastrointestinal Early Cancer Prevention \& Treatment Alliance of China (GECA). Development and validation of a prediction rule for estimating gastric cancer risk in the Chinese high-risk population: a nationwide multicentre study. Gut. 2019;68(9):1576-87. https://doi.org/10.1136/ gutjnl-2018-317556
31. Quach DT, Hiyama T, Gotoda T. Identifying high-risk individuals for gastric cancer surveillance from western and eastern perspectives: Lessons to learn and possibility to develop an integrated approach for daily practice. World J Gastroenterol. 2019;25(27):3546-62. https://doi. org/10.3748/wjg.v25.i27.3546

32. Hamashima C; Systematic Review Group and Guideline Development Group for Gastric Cancer Screening Guidelines. Update version of the Japanese Guidelines for Gastric Cancer Screening. Jpn J Clin Oncol. 2018;48(7):673-83. https://doi.org/10.1093/jjco/hyy077

33. de Vries AC, van Grieken NC, Looman CW, Casparie MK, de Vries E, Meijer GA, Kuipers EJ. Gastric cancer risk in patients with premalignant gastric lesions: a nationwide cohort study in the Netherlands. Gastroenterology. 2008;134(4):945-52. https://doi.org/10.1053/j.gastro.2008.01.071

34. Tatsuta M, Iishi H, Nakaizumi A, Okuda S, Taniguchi $\mathrm{H}$, Hiyama T, Tsukuma H, Oshima A. Fundal atrophic gastritis as a risk factor for gastric cancer. Int J Cancer. 1993;53(1):70-4. https://doi.org/10.1002/ ijc. 2910530114

35. Leung WK, Sung JJ. Review article: intestinal metaplasia and gastric carcinogenesis. Aliment Pharmacol Ther. 2002;16(7):1209-16. https://doi.org/10.1046/j.13652036.2002.01300.x

36. You WC, Li JY, Blot WJ, Chang YS, Jin ML, Gail MH, Zhang L, Liu WD, Ma JL, Hu YR, Mark SD, Correa P, Fraumeni JF Jr, Xu GW. Evolution of precancerous lesions in a rural Chinese population at high risk of gastric cancer. Int J Cancer. 1999;83(5):615-9. https://doi.org/10.1002/ (SICI) 1097-0215(19991126)83:5<615::AIDIJC8>3.0.CO;2-L

37. Rugge M, Meggio A, Pravadelli C, Barbareschi M, Fassan M, Gentilini M, Zorzi M, Pretis G, Graham DY, Genta RM. Gastritis staging in the endoscopic follow-up for the secondary prevention of gastric cancer: a 5-year prospective study of 1755 patients. Gut. 2019;68(1):11-17. https:// doi.org/10.1136/gutjnl-2017-314600

38. Marqués-Lespier JM, González-Pons M, Cruz-Correa M. Current Perspectives on Gastric Cancer. Gastroenterol Clin North Am. 2016;45(3):413-28. https://doi.org/10.1016/j. gtc.2016.04.002

39. Thrift AP, El-Serag HB. Burden of Gastric Cancer. Clin Gastroenterol Hepatol. 2020;18(3):534-542. https://doi. org/10.1016/j.cgh.2019.07.045 